\title{
Thyroid dysfunction secondary to antiviral therapy: a simple management protocol
}

Tariq $\mathrm{S}^{*}$, Bashari WA*, Barry $\mathrm{K}^{\#}$, Ninkovic $\mathrm{M}^{\#}$, Oyibo SO*

*Department of Endocrinology \& "Department of Hepatology, Peterborough City Hospital, Peterborough, UK

\section{Introduction}

Interferon and Ribavarin-based Hepatitis C therapy can induce or exacerbate autoimmune thyroid dysfunction with variable clinical presentation. Being female, having prior thyroid dysfunction and raised anti-thyroid peroxidase (anti-TPO) antibodies are risk factors. We have a protocol for managing thyroid dysfunction that occurs during hepatitis $C$ antiviral therapy. We present four cases.

\section{Case 1}

A 48 year old man diagnosed with hepatitis C infection was found to have mild hypothyroidism with raised anti-TPO antibody levels before starting antiviral therapy. After starting therapy (Pegylated Interferon alfa-2a and Ribavirin) in August 2011, he developed severe hypothyroidism seven months later. He remains on long-term thyroxine replacement.

\section{Case 2}

A 55 year old man diagnosed with hepatitis C infection had a normal thyroid function test (TFT) but raised anti-TPO antibody levels before antiviral treatment. In February 2012 he developed severe hypothyroidism five months after starting antiviral treatment. He remains on long-term thyroxine replacement.

\section{Case 3}

A 41 year old woman diagnosed with hepatitis C infection had a normal TFT and normal antithyroid peroxidase antibody levels before antiviral treatment. In July 2014 she developed thyrotoxicosis six months after starting antiviral treatment. She developed hypothyroidism two months after that and still has mild hypothyroidism.

\section{Case 4}

A 56 year old man diagnosed with hepatitis $C$ infection had a normal TFT before antiviral treatment. In May 2012 he developed thyrotoxicosis four months after starting antiviral treatment. $\mathrm{He}$ then developed hypothyroidism which required thyroxine replacement. This resolved after six months and he is no longer on treatment.

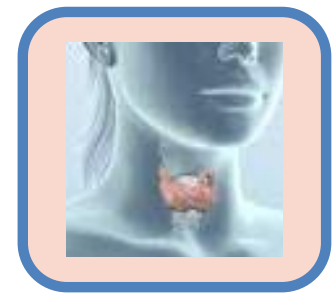

\section{Protocol}

Drug-induced thyroid dysfunction

Thyroid dysfunction secondary to Hepatitis $\mathrm{C}$ or anti-viral therapy

Introduction

The prevalence of thyroid dysfunction in patients using antiviral agents (e.g. interferon for hepatitis $C$ infection) is increasing. The literature quotes $4-20 \%$ depending on the source.

Patients can get:

1. Hypothyroidism

with thyrotoxicosis

3. Graves autoimmune thyrotoxicosis

4. Thyrotoxicosis followed by hypothyroidism

Many patients are asymptomatic and do not require anti-thyroid therapy (e.g., Carbimazole) or thyroxine replacement therapy. Some will recover in a few months or become severely hypothyroid thereafter. The main risk factors for thyroid dysfunction during hep $\mathrm{C}$ infection or its anti-viral therapy are being female and having positive anti-TPO antibodies beforehand. And many occur in first 3-6 months.

Aim of this guide

To help prevent affected patients from undergoing excess testing or starting unnecessary anti-thyroid or thyroxine replacement treatment. This guide also stresses the need for long term surveillance.

Suggestions

All such patients should have TSH and anti-TPO antibodies measured before starting the antiviral therapy.

All such patients should have 3-monthly TSH measurement to catch thyroid disorders, as this is not uncommon in these patients during anti-viral therapy.

3. If patient has abnormal TSH then the lab should automatically check the full thyroid function.

4. Patients with subclinical thyroid disorder (high or low TSH, asymptomatic with normal FT3/FT4) do not need treatment but repeat testing (full test) in 3 months.

. If symptomatic hypothyroidism (high TSH, low FT4) then low-dose levothyroxine $(25-50 \mathrm{mcg}$ daily) may be required but patient must have repeat full test in $4-6$ weeks, then 3 monthly when

6. If symptomatic thyrotoxicosis (low TSH, high FT4) then patient may require beta-blockers, but patient must have a repeat full test in $4-6$ weeks, then 3 monthly if stable.

at risk of developing thyroid dysfunction in the future, so will need yearly screening (TSH-only testing).

You can get advice from the Endocrinologist, especially for those with subclinical or clinical thyrotoxicosis, to assess the need for Carbimazole just in case they have coincidental multinodular or autoimmune thyrotoxicosis. We do not need to create extra appointments for these patients as telephon follow-up (telemedicine) will suffice for most of them.

Pathology request form

Additionally, accurate clinical details are essential when requesting the tests.

When requesting TSH \& anti-TPO antibodies for pre-treatment screening, one must put 'viral

disease/on anti-viral therapy at risk of thyroid disorder" e.g.
When requesting TSH for follow-up, one must put "viral disease/on anti-viral therapy at risk of thyroid disorder" e.g.

When requesting full TFT in patients with abnormal TSH levels, one must put 'viral or druginduced thyroid disorder" e.9.

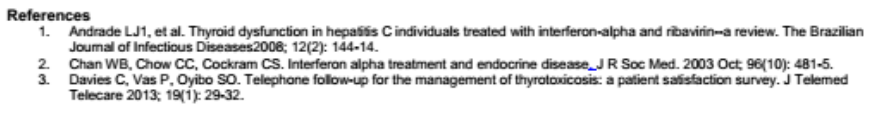

\section{Conclusion}

Our protocol ensures that patients with hepatitis $\mathrm{C}$ infection have their thyroid hormones and anti-TPO antibody levels measured prior to starting antiviral therapy. Thyrotoxicosis is monitored and does not require anti-thyroid treatment. Hypothyroidism is treated if severe or there are symptoms. All patients are followed up after antiviral treatment. 